\title{
手性锑 $(\mathrm{V})$ 阴/阳离子对催化的不对称转移氢化反应
}

\author{
冯向青 $a, b$ 杜海峰*,a,b \\ ( ${ }^{a}$ 中国科学院化学研究所 北京国家分子科学研究中心 中国科学院分子识别与功能重点实验室 北京 100190) \\ $(b$ 中国科学院大学 北京 100049)
}

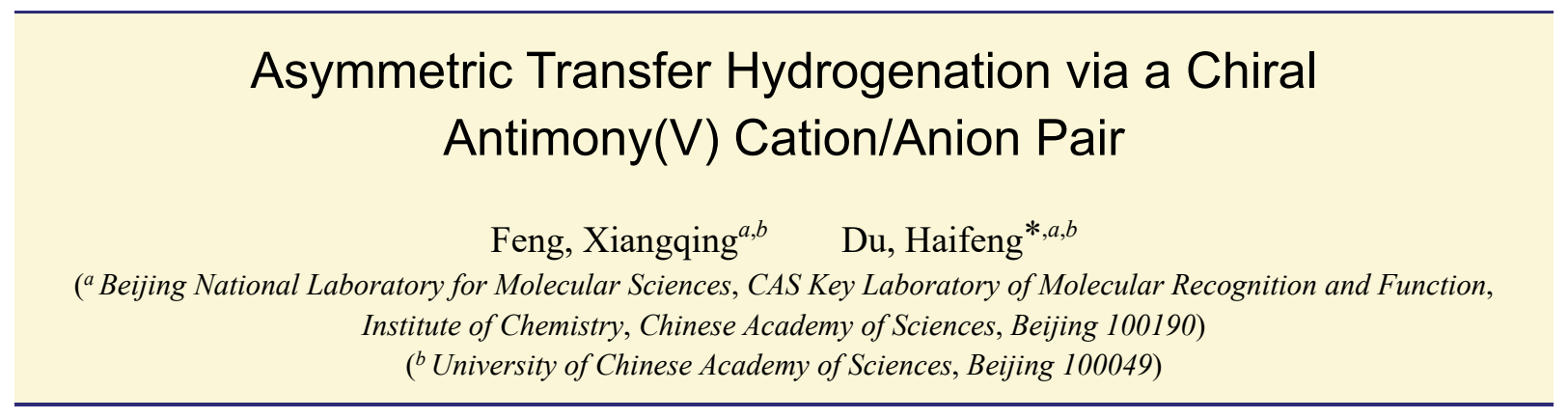

Pnictogen 键是氮族正静电势和受体上负静电势之 间的非共价相互作用 ${ }^{[1-2]}$, 这种相互作用在晶体结构、超 分子组装以及阴离子识别等领域很常见, 然而在合成化 学中的应用研究较少. Pnictogen 键催化 ${ }^{[3]}$ 可以实现不同 于传统 Lewis 酸催化的反应性, 为发展新型的催化机理 提供了可能. 近几年 Pnictogen 键催化取得了一些进展 (图 1) ${ }^{[4-5]}$, 如篗卤反应、环氧开环反应和转移氢化反应 等, 但是将其用于不对称催化的反应目前还没有报道. 近日, 南方科技大学化学系谭斌课题组 ${ }^{[6]}$ 在该领域取得 了突破性的进展, 该研究团队使用手性锑阴/阳离子催 化剂实现了苯并噁嗪衍生物的不对称转移氢化反应.
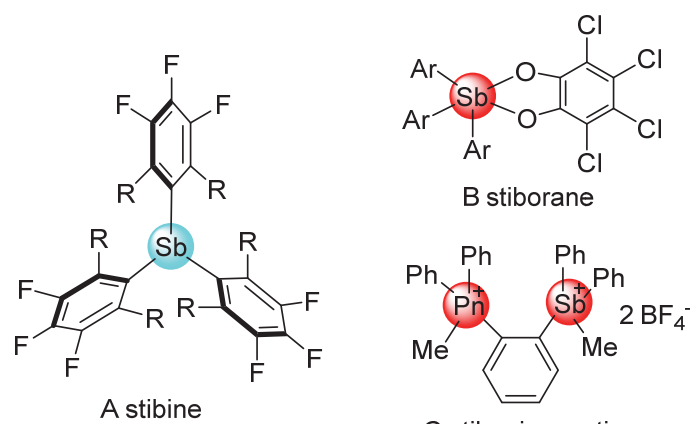

C stibonium cation

图 1 Pnictogen 键催化剂

Figure 1 Pnictogen-bonding catalysts

首先, 作者以手性扁桃酸衍生物作为手性来源, 合 成得到了一系列的手性 $\mathrm{Sb}(\mathrm{V})$ 化合物 3 (Scheme 1). 将其
用于 Hantzsch 酯为氢源的苯并噁嗪的转移氢化反应中, 发现纯化后的 3 的反应活性明显降低，但是对映选择性 基本没有影响. 进一步研究发现, 反应过程中实际反应 活性物种是手性锑 $(\mathrm{V})$ 阴/阳离子对 4 , 通过配体的䇻选 对比和反应条件的考察, 对苯并噁嗪的不对称转移氢化 反应可以高效高选择性地实现.

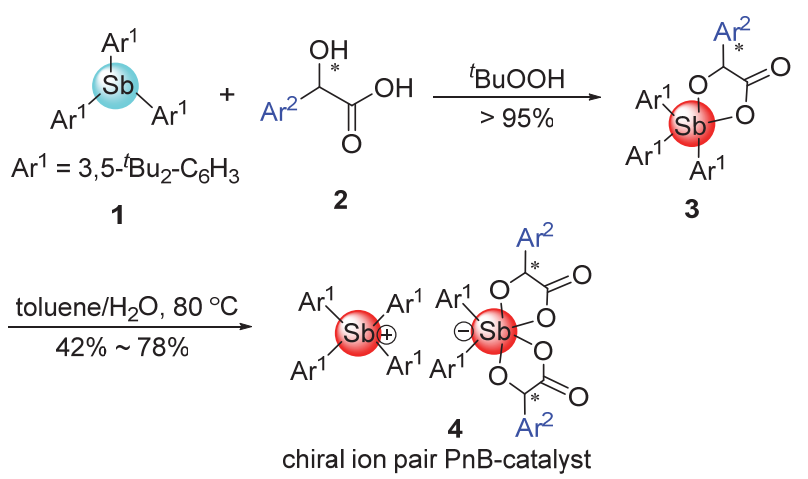

图式 1 手性 PnB-催化剂的合成

Scheme 1 Synthesis of chiral PnB-catalyst

在得到最佳条件后, 作者通过篮选底物范围考察了 反应的普适性(Scheme 2). 对 6-, 7-位具有给电子或吸电 子取代基的苯并噁嗪，反应可以以良好的产率(93\% 99\%)和优秀的对映选择性(95\% 98\%)得到相应的产物， 更大的取代基如叔丁基等也具有很好的耐受性. 带有扩 展 $\pi$ 系统的菜并噁嗪也可以得到 $99 \%$ 的产率和高达 $95 \%$ ee. 3-位带有不同取代芳基的底物也可以以 $92 \%$ 的对映

* Corresponding author. E-mail: haifengdu@iccas.ac.cn. Published online June 19, 2021. 
选择性定量地转化为相应产物, 但是反应需要较长的时 间. 对于 2,2-二甲基取代苯并噁嗪，反应可以实现定量 转化得到 $94 \% \mathrm{ee}$, 而其五元类似物展现出较差的对映 选择性 $(85 \% e e)$. 作为比较, 作者还尝试了活性较低的 2-苯基喹啉和苯并噁嗪酮, 反应可以得到良好的收率和 $89 \%$ 的对映选择性.
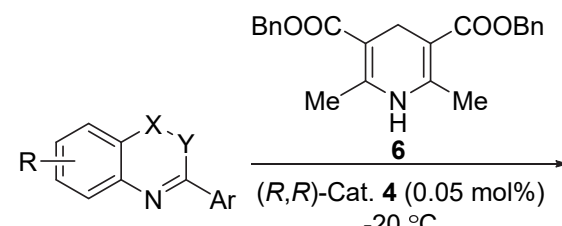
$-20^{\circ} \mathrm{C}$

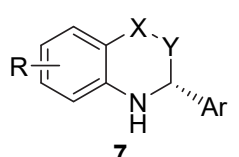<smiles>[R]c1ccc2c(c1)N[C@H](c1ccccc1)CO2</smiles>

$\mathrm{R}=\mathrm{OMe}, 99 \%(97 \%$ ee $), 12 \mathrm{~h}$

$\mathrm{R}=\mathrm{Br}, 99 \%$ (98\% ee), $24 \mathrm{~h}$

$\mathrm{R}=\mathrm{NO}_{2}, 93 \%$ (98\% ee), $12 \mathrm{~d}$

$\mathrm{R}={ }^{t} \mathrm{Bu}, 99 \%$ (95\% ee), $24 \mathrm{~h}$<smiles>[R]c1ccc2c(c1)OC[C@@H](c1ccccc1)N2</smiles>

$\mathrm{R}=\mathrm{F}, 96 \%(95 \%$ ee $), 48 \mathrm{~h}$ $\mathrm{R}=\mathrm{COOMe}, 99 \%(95 \%$ ee $), 72 \mathrm{~h}$<smiles>c1ccc([C@@H]2COc3cc4ccccc4cc3N2)cc1</smiles>

$99 \%(95 \%$ ee) $60 \mathrm{~h}$

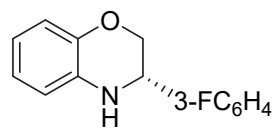
$99 \%(92 \%$ ee $)$ $60 \mathrm{~h}$<smiles>O=C1Oc2ccccc2N[C@H]1c1ccccc1</smiles>
$87 \%(89 \%$ ee) r.t., $48 \mathrm{~h}$<smiles>CC1(C)Oc2ccccc2N[C@H]1c1ccccc1</smiles>
$99 \%(94 \%$ ee $)$ $24 \mathrm{~h}$<smiles>CC1(C)c2ccccc2N[C@H]1c1ccccc1</smiles>
$12 \mathrm{~h}$<smiles>c1ccc([C@H]2CCc3ccccc3N2)cc1</smiles>

$99 \%(89 \%$ ee $)$ r.t., $36 \mathrm{~h}$
图式 2 反应的普适性考察

Scheme 2 Scope of the substrates

随后, 作者对反应机理进行了研究, 通过时间反应 和对照控制实验(表 1), 探索了催化剂阴/阳离子的作用. 在不加入任何催化剂的情况下, 可以观察到缓慢的背景 反应. 为排除扁桃酸是活性催化物种的可能性, 使用 $(R)$-2-氯扁桃酸 $(\mathbf{2} \mathbf{a})$ 作为催化剂进行尝试, 反应虽然表 现出很高的活性, 但需要较长的反应时间, 而且只能得 到 12\%的对映选择性. 四芳基溴化锑 8 和四芳基锑扁桃 酸盐 9 都没有催化活性, 而使用 $\left[\mathrm{Sb}\left(\mathrm{Ar}^{t \mathrm{Bu}}\right)_{4}\right]^{+}\left[\mathrm{B}\left(\mathrm{Ar}^{\mathrm{F}}\right)_{4}\right]$ (10)作为催化剂时, 反应活性明显提高, 这证实了锑阳 离子的催化活性.
表 1 控制实验

Table 1 Control experiments

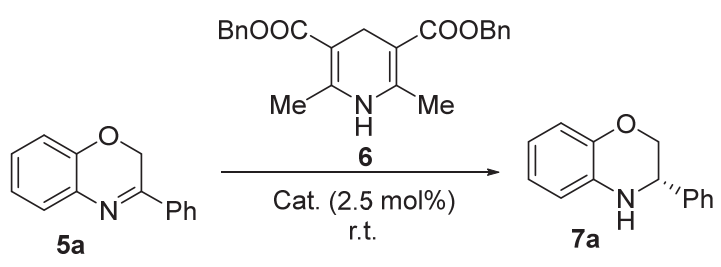

$\mathrm{C}_{6} \mathrm{H}_{4} \mathrm{Cl}-2$

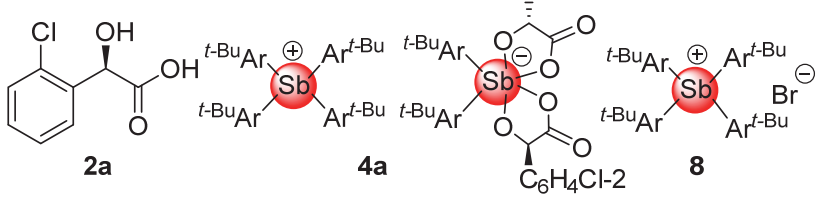<smiles>[14CH3]OC(=O)C(O)c1ccccc1</smiles>

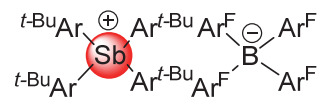

\begin{tabular}{cccccc}
\hline Entry & Cat. & Solvent & Time $/ \mathrm{h}$ & Yield/\% & $e e / \%$ \\
\hline 1 & $\mathbf{2 a}$ & Toluene & 12 & 99 & 12 \\
2 & $\mathbf{4 a}$ & Toluene & 2 & 99 & 82 \\
4 & $\mathbf{9}$ & $\mathrm{C}_{6} \mathrm{D}_{6}$ & 12 & $<5$ & - \\
\hline
\end{tabular}

综上所述，谭斌课题组实现了首例由手性锑离子对 促成的不对称 pnictogen 键催化. 使用价格低廉、来源丰 富的扁桃酸类似物作为手性配体进行不对称诱导. 这类 新开发的手性 $\mathrm{PnB}$ 催化剂在不对称转移氢化反应中表 现出优良的性能，反应条件温和，使用较低的催化剂用 量，可以得到优秀的对映选择性和反应活性，对不同取 代基的底物都有很好的兼容性. 作者还进行了机理研 究，观察到配体交换现象，控制实验表明锑阴/阳离子对 保证反应的高活性和高选择性是缺一不可的. 锑阳离子 具有更深的 $\sigma$-hole，可能是该手性 PnB 催化剂高性能的 原因，锑抗衡阴离子具有路易斯碱性，体积大且柔软， 是 $\mathrm{PnB}$ 催化剂高活性的保障.

\section{References}

[1] Girolami, G. S. J. Chem. Educ. 2009, 86, 1200.

[2] Scheiner, S. Acc. Chem. Res. 2013, 46, 280.

[3] Gini, A.; Paraja, M.; Galmés, B.; Besnard, C.; Poblador-Bahamonde, A. I.; Sakai, N.; Frontera, A.; Matile, S. Chem. Sci. 2020, 11, 7086.

[4] Bayne, J. M.; Stephan, D. W. Chem. Soc. Rev. 2016, 45, 765.

[5] Yang, M.; Hirai, M.; Gabbaï, F. P. Dalton Trans. 2019, 48, 6685.

[6] Zhang, J.; Wei, J.; Ding, W.-Y.; Li, S.; Xiang, S.-H.; Tan, B. J. Am. Chem. Soc. 2021, 143, 6382. 